\title{
PIK3CA gene mutation associated with poor prognosis of lung adenocarcinoma
}

This article was published in the following Dove Press journal:

OncoTargets and Therapy

3 May 2013

Number of times this article has been viewed

\section{Lina Zhangl,* \\ Lei $\mathrm{Shi}^{2}$,* \\ Xiaoting Zhao' \\ Yue Wang' \\ Wentao Yue' \\ 'Department of Molecular and Cellular Biology, Beijing Chest Hospital, Capital Medical University, Beijing TB and Thoracic Tumor Research Institute, Beijing, People's Republic of China; ${ }^{2}$ Department of Hepatobiliary Surgery, the First Affiliated Hospital Medical School of Xi'an Jiaotong University, Xi'an, People's Republic of China \\ *These authors contributed equally to this work}

Correspondence: Wentao Yue Department of Cellular and Molecular Biology, Beijing Chest Hospital, Capital Medical University, 97 Beimachang, Tongzhou, Beijing, People's Republic of China 101149

Tel +861089509373

$\mathrm{Fax}+86 \quad 1080507349$

Email yuewt2000@yahoo.com
Purpose: PIK3CA gene mutations have been detected in many malignancies, but the frequency of different mutations and their role in the carcinogenesis of lung adenocarcinoma are still unclear. The purpose of this study was to explore the clinical pathological impact and prognostic implications of PIK3CA mutations in lung adenocarcinoma.

Methods: Five common PIK3CA mutations (E542K, E545K, and E545D mutation in exon 9, H1047R and H1047L mutation in exon 20) were detected by amplification refractory mutation system (ARMS) allele-specific polymerase chain reaction (PCR), in 122 patients with lung adenocarcinoma. The relationships were studied between these mutations and various clinicopathologic variables (age, lymph node status, distant metastasis, clinicopathologic stage, smoking status, and progression-free survival).

Results: In total, 25 mutations were identified, of which 24 mutations were clustered in exon 20, and one mutation in exon 9. The most common mutations were H1047R (18 out of the 122 patients, $14.8 \%$ ) in exon 20. PIK3CA-mutated tumors were more frequently found in patients with lymph node positive metastasis status $(P<0.05)$. There was no significant association between PIK3CA mutations and age, distant metastasis, smoking status, or clinicopathologic stage. However, mutations were found less frequently in the early clinicopathologic stage patients ( six in 50 cases, 12\%) than in advanced stage (19 in 72 cases, 26.4\%). Higher frequency of H1047R mutations was associated with poor prognosis, and this association reached statistical significance $(P<0.05)$.

Conclusion: Our data indicate that the PIK3CA mutations H1047R and H1047L are significant genetic alterations in lung adenocarcinoma. Among lung adenocarcinoma patients who underwent curative resection, PIK3CA mutations were associated with shorter progression-free survival. Our findings demonstrated a significant role of $P I K 3 C A$ in lung adenocarcinoma.

Keywords: Phosphatidylinositol-3-kinase catalytic subunit (PIK3CA); H1047R mutation; Cancer; Lung neoplasms

\section{Introduction}

Non-small-cell lung cancer (NSCLC) is the leading cause of cancer mortality worldwide. Lung adenocarcinoma is the main type of NSCLC. ${ }^{1}$ Owing to its complex tumorigenesis, lung adenocarcinoma is exceedingly difficult to treat and prognosticate. Recently, in order to find therapeutic targets and prognostic biomarkers, research on lung adenocarcinoma has focused on gene mutations.

The activation of the phosphatidylinositol 3-kinase (PI3K)/AKT pathway is thought to play a critical role in the development of lung cancer. ${ }^{2}$ As in most cancers, during the development of lung adenocarcinoma, genetic defects accumulate, resulting in 
the altered activity of the PI3K/AKT signaling pathway. The PIK3CA gene encodes the catalytic p110- $\alpha$ subunit of PI3K. ${ }^{3}$ It has been suggested that $P I K 3 C A$ is somatically mutated in lung cancers. ${ }^{4}$ Most of the previous studies investigated the PIK3CA mutations using gene sequencing technology, and the positive mutation rate was less than $10 \%$ in lung cancer. ${ }^{5,6}$ Recently, the research of Chaft et al detected four types of PIK3CA mutations (E545K, E542K, H1047R, and $\mathrm{H} 1047 \mathrm{~L}$ ) in lung adenocarcinoma; however, the rate of mutation was only $2 \%$ by mass spectrometry-based nucleic acid assay technology. ${ }^{7}$ It was unclear about the relation between PIK3CA mutations and patient prognosis. We need more research to understand the impact of $P I K 3 C A$ mutations in lung adenocarcinoma. Therefore, we attempted to detect the frequency of PIK3CA mutations using a new method - amplification refractory mutation system (ARMS) allelespecific polymerase chain reaction (PCR) - and also assessed the clinicopathologic significance and prognosis of $P I K 3 C A$ mutation status, in a large series of lung adenocarcinoma. ${ }^{8}$

The aim of this study was to identify significantly mutated loci not previously associated with lung adenocarcinoma and to describe the relationships between PIK3CA mutations and clinical features. Moreover, our integration of ARMS-PCR and mutation data provides a novel view of $P I K 3 C A$ alterations in lung adenocarcinoma. These findings further our understanding of lung adenocarcinoma and provide clues to prognosis and new therapeutic targets.

\section{Materials and methods}

\section{Patients and tissue samples}

The analysis was conducted in 122 patients with histologically confirmed primary lung adenocarcinoma who underwent surgical resection between April 2008 and September 2010 at the Beijing Chest Hospital, Capital Medical University, Beijing, People's Republic of China. All patients gave informed consent according to a study protocol that was approved by Beijing Chest Hospital Human Tissue Committee and Research Ethics Board (REB). All tissue samples had been flash frozen in liquid nitrogen within 30 minutes of surgery and stored at $-80^{\circ} \mathrm{C}$. The age of the studied patients ranged from 36 to 83 years with a median of 61.5 years. The histological classification was based on the 2004 World Health Organization criteria. Tumors were staged according to the 2009 Union for International Cancer Control guidelines. The detailed clinical characteristics of the 122 primary lung adenocarcinoma patients are listed in Table 1.
Table I Clinical characteristics of I 22 patients

\begin{tabular}{|c|c|c|c|c|}
\hline & \multirow[t]{2}{*}{ Total } & \multicolumn{2}{|l|}{ Sex } & \multirow[t]{2}{*}{$\boldsymbol{P}$} \\
\hline & & Male & Female & \\
\hline Number of patients & 122 & 72 & 50 & \\
\hline \multicolumn{5}{|l|}{ Age (years) } \\
\hline $0-60$ & 60 & 31 & 29 & 0.10 \\
\hline$>60$ & 62 & 41 & 21 & \\
\hline \multicolumn{5}{|l|}{ Lymph node status } \\
\hline LN (-) & 50 & 34 & 18 & 0.22 \\
\hline LN (+) & 72 & 38 & 32 & \\
\hline \multicolumn{5}{|l|}{ Distant metastasis } \\
\hline$M(-)$ & III & 64 & 47 & 0.52 \\
\hline$M(+)$ & II & 8 & 3 & \\
\hline \multicolumn{5}{|c|}{ Clinicopathologic stage } \\
\hline I-II & 50 & 30 & 20 & 0.85 \\
\hline III-IV & 72 & 42 & 30 & \\
\hline \multicolumn{5}{|l|}{ Smoking status } \\
\hline Smoker & 59 & 55 & 4 & $0.000^{\mathrm{a}}$ \\
\hline Nonsmoker & 60 & 16 & 44 & \\
\hline Missing & 3 & I & 2 & \\
\hline
\end{tabular}

Note: aSmoking status is significantly associated with gender. Abbreviations: LN, lymph node; M, metastasis.

\section{DNA extraction}

The DNA was extracted from the frozen tissues using the TIANamp Genomic DNA Kit (Tiangen Biotech, Beijing, People's Republic of China), according to the manufacturer's protocol. The DNA was qualitatively assessed by agarose gel electrophoresis and was quantified spectrophotometrically, to confirm that A260/A230 value is greater than 2.0 and A260/A280 value between 1.8 and 2.0 .

\section{Detection of PIK3CA mutations}

For the ARMS-PCR analysis of the five common PIK3CA mutations (E542K, E545K, and E545D mutation in exon 9, H1047R and H1047L mutation in exon 20), samples were loaded, in triplicate, into 96-well reaction plates, using the AmoyDx ${ }^{\mathrm{TM}}$ PIK3CA Five Mutation Detection Kit (Amoy Diagnostics Co, Ltd, Xiamen, People's Republic of China), according to the manufacturer's instructions, for each sample. The mutation assay for each sample and control assay ( $P I K 3 C A$ mixed standard and no-template controls) were analyzed in the same PCR run, to avoid run-to-run variations in threshold settings. The assays were performed with the Applied Biosystems 7500 Real-Time PCR System (Life Technologies, Carlsbad, CA, USA). Predenaturing was carried out at $95^{\circ} \mathrm{C}$ for 5 minutes, followed by 15 cycles of denaturing at $95^{\circ} \mathrm{C}$ for 25 seconds, $64^{\circ} \mathrm{C}$ for 20 seconds, and $72^{\circ} \mathrm{C}$ for 20 seconds, in order to reach a full plateau for 
all samples, and was followed by the detection stage with 20 cycles at $93^{\circ} \mathrm{C}$ for 25 seconds, $60^{\circ} \mathrm{C}$ for 35 seconds, and $72^{\circ} \mathrm{C}$ for 20 seconds. The analysis of the target mutations in the samples was accomplished by measuring the fractional cycle number at which the degree of expression reached a fixed cycle threshold $\left(\mathrm{C}_{\mathrm{T}}\right)$. Based on different mutant $\mathrm{C}_{\mathrm{T}}$ values, the detection results were categorized as either positive or negative for mutation.

\section{Statistical analysis}

Chi-square and the Fisher's exact test were used to analyze the association of mutations with clinical characteristics. The Kaplan-Meier method was used to estimate survival and progress free survival, and the exact log rank test was used to compare differences in survival the distributions among groups. All data were analyzed by statistical software SPSS 16.0 (SPSS Inc, Chicago, IL, USA). A $P$-value less than 0.05 was considered to be statistically significant.

\section{Results}

\section{PIK3CA mutations were found}

\section{in lung adenocarcinoma}

Among the 122 patients who underwent curative resection of stage I to IV lung adenocarcinoma, we detected PIK3CA mutations in exons 9 and 20, using ARMS-PCR technology.
In all, 24 patients $(19.7 \%)$ harbored at least one mutation in the regions analyzed. A total of 25 point mutations were found in these 24 patients: one mutation occurred in the helical domain (E542K, exon 9), and 24 mutations were in the kinase domain (18 of these were mutations of H1047R and six were of H1047L, exon 20). One patient carried two mutations in exon 20 (H1047R and H1047L). The "hot spot" was H1047R, located in the kinase domain (exon 20). None of the patients showed mutations in both exon 9 and exon 20. The frequency and distribution of the mutations are shown in Table 2.

\section{PIK3CA mutations correlated with lymph node (LN) status and tumor stage}

We analyzed the clinical characteristics of the lung adenocarcinoma cases according to $P I K 3 C A$ mutation status (Tables 2 and 3). Compared with $P I K 3 C A$ wild-type tumors, $P I K 3 C A$ mutations were found more frequently in tumors with LN metastasis (LN [+]), 20 point mutations in LN (+), and five point mutations in LN patients without LN metastasis $(\mathrm{LN}[-])(P=0.029)$. PIK3CA mutations occurred in $12 \%(6 / 50)$ of patients at early clinicopathologic stage (I-II) and in $26.4 \%(19 / 72)$ of patients at advanced stage (III-IV) disease, but the association was not statistically significant $(P=0.122)$. PIK3CA mutations were not associated with

Table 2 Frequency of each PIK3CA mutation type in lung adenocarcinoma

\begin{tabular}{|c|c|c|c|c|c|c|c|c|c|c|c|}
\hline \multirow[t]{3}{*}{ Exon } & \multirow{3}{*}{$\begin{array}{l}\text { Total } \\
\text { (\%) }\end{array}$} & \multicolumn{4}{|l|}{ Exon 20} & \multicolumn{6}{|c|}{ Exon 9} \\
\hline & & \multicolumn{2}{|l|}{ HIO47R } & \multicolumn{2}{|l|}{ HI047L } & \multicolumn{2}{|c|}{ E542K } & \multicolumn{2}{|c|}{ E545K } & \multicolumn{2}{|c|}{ E545D } \\
\hline & & $\begin{array}{l}\text { Male } \\
(\%)\end{array}$ & $\begin{array}{l}\text { Female } \\
(\%)\end{array}$ & $\begin{array}{l}\text { Male } \\
(\%)\end{array}$ & $\begin{array}{l}\text { Female } \\
(\%)\end{array}$ & $\begin{array}{l}\text { Male } \\
(\%)\end{array}$ & $\begin{array}{l}\text { Female } \\
(\%)\end{array}$ & $\begin{array}{l}\text { Male } \\
(\%)\end{array}$ & $\begin{array}{l}\text { Female } \\
(\%)\end{array}$ & $\begin{array}{l}\text { Male } \\
(\%)\end{array}$ & $\begin{array}{l}\text { Female } \\
(\%)\end{array}$ \\
\hline Number of mutations & $25(20.5 \%)^{a}$ & $10(8.2 \%)$ & $8(6.6 \%)$ & $3(2.5 \%)$ & $3(2.5 \%)$ & 0 & 0 & 0 & I (0.8\%) & 0 & 0 \\
\hline Age (years) & & & & & & 0 & 0 & 0 & I (0.8\%) & 0 & 0 \\
\hline $0-60$ & 15 (I2.3\%) & $5(4.1 \%)$ & $6(4.9 \%)$ & $3(2.5 \%)$ & 0 & 0 & 0 & 0 & 0 & 0 & 0 \\
\hline$>60$ & 10 (8.2\%) & $5(4.1 \%)$ & $2(1.6 \%)$ & 0 & $3(2.5 \%)$ & 0 & 0 & 0 & 0 & 0 & 0 \\
\hline \multicolumn{12}{|l|}{ Lymph node status } \\
\hline LN (-) & $5(4.1 \%)$ & I (0.8\%) & $3(2.5 \%)$ & I (0.8\%) & 0 & 0 & 0 & 0 & 0 & 0 & 0 \\
\hline LN $(+)$ & $20(16.4 \%)^{b}$ & $9(7.4 \%)$ & $5(4.1 \%)$ & $2(1.6 \%)$ & $3(2.5 \%)$ & 0 & 0 & 0 & I (0.8\%) & 0 & 0 \\
\hline \multicolumn{12}{|l|}{ Distant metastasis } \\
\hline$M(-)$ & $22(18 \%)$ & $9(7.4 \%)$ & $7(5.7 \%)$ & $2(1.6 \%)$ & $3(2.5 \%)$ & 0 & 0 & 0 & I (0.8\%) & 0 & 0 \\
\hline$M(+)$ & $3(2.5 \%)$ & I (0.8\%) & I (0.8\%) & I (0.8\%) & 0 & 0 & 0 & 0 & 0 & 0 & 0 \\
\hline \multicolumn{12}{|l|}{ Clinicopathologic stage } \\
\hline I-II & $6(4.9 \%)$ & $2(1.6 \%)$ & $3(2.5 \%)$ & I (0.8\%) & 0 & 0 & 0 & 0 & 0 & 0 & 0 \\
\hline III-IV & $19(15.6 \%)$ & $8(6.6 \%)$ & $5(4.1 \%)$ & $2(1.6 \%)$ & $3(2.5 \%)$ & 0 & 0 & 0 & I (0.8\%) & 0 & 0 \\
\hline \multicolumn{12}{|l|}{ Smoking status } \\
\hline Smoker & $13(10.7 \%)$ & $9(7.4 \%)$ & 0 & $3(2.5 \%)$ & I (0.8\%) & 0 & 0 & 0 & 0 & 0 & 0 \\
\hline Nonsmoker & II (9.0\%) & 0 & $8(6.6 \%)$ & 0 & $2(1.6 \%)$ & 0 & 0 & 0 & I (0.8\%) & 0 & 0 \\
\hline Missing & $\mathrm{I}(0.8 \%)$ & I (0.8\%) & 0 & 0 & 0 & 0 & 0 & 0 & 0 & 0 & 0 \\
\hline
\end{tabular}

Notes: a One sample had both mutations of HI047R and HI047L; ${ }^{\mathrm{b} P}<0.05$.

Abbreviations: LN, lymph node; $M$, metastasis. 
Table 3 Clinical characteristics according to PIK3CA mutation status in lung adenocarcinoma

\begin{tabular}{|c|c|c|c|c|}
\hline & \multirow{2}{*}{$\begin{array}{l}\text { Total } \\
(n=I 22) \\
N(\%)\end{array}$} & \multicolumn{2}{|c|}{ PIK3CA mutation status } & \multirow[t]{2}{*}{$P^{a}$} \\
\hline & & $\begin{array}{l}\text { Wild-type } \\
(n=98) \\
N(\%)\end{array}$ & $\begin{array}{l}\text { Mutant } \\
(n=24) \\
N(\%)\end{array}$ & \\
\hline \multicolumn{5}{|l|}{ Age (years) } \\
\hline Mean (range) & $60(36-83)$ & 61 (37-83) & $58(36-73)$ & \\
\hline $0-60$ & $60(49 \%)$ & $46(47 \%)$ & $14(58 \%)$ & 0.32 \\
\hline$>60$ & $62(51 \%)$ & $52(53 \%)$ & 10 (42\%) & \\
\hline \multicolumn{5}{|c|}{ Lymph node status } \\
\hline LN (-) & $52(43 \%)$ & 47 (48\%) & $5(21 \%)$ & 0.029 \\
\hline $\operatorname{LN}(+)$ & $70(57 \%)$ & $5 \mathrm{I}(52 \%)$ & 19 (79\%) & \\
\hline \multicolumn{5}{|c|}{ Distant metastasis } \\
\hline$M(-)$ & III (9I\%) & 89 (91\%) & $22(92 \%)$ & 0.79 \\
\hline$M(+)$ & II (9\%) & $9(9 \%)$ & $2(8 \%)$ & \\
\hline \multicolumn{5}{|c|}{ Clinicopathologic stage } \\
\hline I-II & $50(41 \%)$ & $44(45 \%)$ & $6(25 \%)$ & 0.122 \\
\hline III-IV & $72(59 \%)$ & $54(55 \%)$ & I 8 (75\%) & \\
\hline \multicolumn{5}{|l|}{ Smoking status } \\
\hline Smoker & $59(48 \%)$ & 47 (48\%) & $12(50 \%)$ & 0.78 \\
\hline Nonsmoker & $60(49 \%)$ & $49(50 \%)$ & II (46\%) & \\
\hline Missing & $3(2 \%)$ & $2(2 \%)$ & I (4\%) & \\
\hline
\end{tabular}

Notes: ${ }^{\mathrm{a}} \chi^{2}$ test used for binary/categorical variables; ${ }^{\mathrm{b} P}<0.05$.

Abbreviations: LN, lymph node; $M$, metastasis.

smoking status in the total group of patients $(P=0.78)$. No correlation was identified between the PIK3CA mutation rate and distant metastasis, age, or gender.

\section{PIK3CA mutations and patient progression-free survival (PFS)}

We assessed the influence of PIK3CA mutation on patient PFS in resectable lung adenocarcinoma. The median follow up for the cohort was 19 months (0-47 months). Patients harboring PIK3CA-mutated lung adenocarcinoma showed a marginally significant shorter median PFS of 12 months (0-42 months) compared with patients with PIK3CA wildtype tumors, for whom median PFS was about 20 months (1-47 months). The patients with PIK3CA-mutated tumors experienced an increase in cancer-specific mortality, although statistical significance was not reached $(P=0.385)$ (Figure 1A). We also assessed the five PIK3CA mutation types separately. In our study, the H1047R mutant is the most common mutation. H1047R mutations associated with survival, and reached statistical significance $(P=0.032)$ (Figure 1B); the median PFS of H1047R (+) patients was 9 months (95\% confidence interval [CI] 7.2-10.8), far under the median PFS of 26 months (95\% CI 17.2-36.8) for H1047R (-) patients.
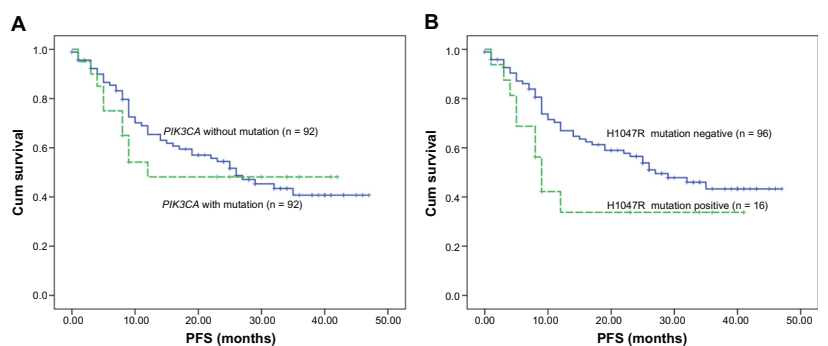

Figure I Kaplan-Meier curves for PFS in lung adenocarcinoma with five PIK3CA mutations (A) and for the HI047R mutation type (B).

Notes: In (A), lung a denocarcinoma patients harboring PIK3CA with mutation $(n=20)$ showed a marginally significant shorter median PFS of 12 months (0-42 months) compared with 20 months ( $1-47$ months) in patients without mutation $(n=92)$. The patients with PIK3CA-mutated tumors experienced an increase in cancer-specific mortality, although statistical significance was not reached $(P=0.385)$. In $(B)$, the median PFS of HI047R mutation-positive patients $(n=16)$ was 9 months $(95 \% \mathrm{Cl}$ 7.2-10.8), far under the median PFS of 26 months (95\% Cl 17.2-36.8) for HIO47R mutation-negative patients $(n=96)$. HI047R mutations associated with survival, and reached statistical significance $(P=0.032)$. When the mutation of HI047R was included, the association reached statistical significance $(P=0.032)$.

Abbreviations: $\mathrm{Cl}$, confidence interval; cum, cumulative; PFS, progression-free survival.

\section{Discussion}

Most of the reported PIK3CA mutations were localized at hotspots in exon 9 and exon 20 of the gene. Exon 20 encodes the catalytic domain of $\mathrm{p} 110 \alpha$, and mutations in this domain may constitutively activate its enzymatic activity. Exon 9 encodes the helical domain of $\mathrm{p} 110 \alpha$, and those mutations depress an inhibitory interaction between the N-terminal Src homology 2 (SH2) domain of $\mathrm{p} 85$ and the p110 $\alpha$ catalytic subunit. Expression of the PIK3CA mutations leads to increased oncogenic potential in vitro and in vivo. Direct gene sequencing has been commonly used to analyze mutations of the PIK3CA gene. However, this method was unable to detect the presence of mutant $P I K 3 C A$ when present at $<30 \%$ of the total. ${ }^{8}$ The ARMS-PCR assay, a new detection method, identified more mutations in the clinical samples than did direct sequencing. The ARMS-PCR assays were able to detect the presence of mutations at $1 \%$ of the total. These assays were more sensitive than sequencing and could detect five copies of mutant DNA in proportions as low as $0.1 \%$ of the total DNA. ${ }^{8}$ In our study, we tested the five "hotspot" mutations (E542K, E545K, E545D mutation in exon 9, H1047R and H1047L mutation in exon 20) in 122 lung adenocarcinomas using ARMS-PCR; we found that the positive rate $(19.7 \%)$ was much higher than in early reports studies. ${ }^{7,9,10}$

The PIK3CA mutations found in human cancers primarily occur at two "hot spots:" E545K in the helical domain and H1047R in the catalytic domain., ${ }^{71,12}$ These mutations are known to promote the catalytic activity of $\mathrm{p} 110 \alpha$, thereby leading to constitutive activation of the PI3K 
signaling pathway. ${ }^{13}$ Research by Engelman et al indicated that transgenic mice with lung-specific induction of the kinasedomain mutant p110 $\alpha$ H1047R (in exon 20) developed lung adenocarcinoma. ${ }^{14}$ Yamaguchi et al determined that E545K and H1047R mutations in $\mathrm{p} 110 \alpha$ enhanced invadopodiamediated extracellular matrix degradation and invasion. ${ }^{15}$ This finding provides mechanistic insight into the role of $\mathrm{p} 110 \alpha$ mutations in cancer invasion, and Yamaguchi suggested that PI3K signaling, via $\mathrm{p} 110 \alpha$, regulates invadopodia-mediated invasion and migration of breast cancer cells. ${ }^{15}$ In this study, we analyzed the clinicopathologic significance of five "hotspot" PIK3CA mutations among 122 lung adenocarcinoma patients. The data showed that PIK3CA mutations were associated with LN metastasis. Lung adenocarcinoma patients with LN (+) had many more mutations (20 in 70 cases, 28.6\%) than did patients with LN (-) (five in 52 cases, 9.6\%) $(P=0.029)$. Moreover, there was no significant association between $P I K 3 C A$ mutations and age $(P=0.32)$, distant metastasis $(P=0.79)$, smoking status $(P=0.78)$, or clinicopathologic stage $(P=0.122)$, by Chi-square test. However, mutations were found less frequently in the early clinicopathologic stage patients ( $\mathrm{six}$ in 50 cases, 12\%) than in advanced stage (19 in 72 cases, $26.4 \%)$.

PIK3CA mutations activate AKT through phosphorylation, and pAKT expression has been reported to be associated with poor prognosis. ${ }^{15}$ Some studies have shown that $P I K 3 C A$ mutation predicts shorter survival in colorectal and breast cancers. ${ }^{16-21}$ Because the effect of PIK3CA mutations on lung adenocarcinoma patient prognosis has rarely been studied, we investigated the prognostic significance of $P I K 3 C A$ mutations in the present study. In our research, we examined on prognostic significance of five hot spot $P I K 3 C A$ mutations in 122 lung adenocarcinoma patients, focusing on PFS. In our study, the H1047R mutant is the most common mutation. H1047R mutations associated with survival, and reached statistical significance $(P=0.032)$ (Figure 1B). The median PFS of H1047R (+) patients was 9 months $(95 \%$ CI 7.2-10.8), which was far under that of H1047R (-) patients, who had a mean PFS of 26 months (95\% CI 17.2-36.8). In our cohorts, the data on cancer treatment were limited. Nonetheless, it is unlikely that chemotherapy use differed according to tumor PIK3CA-mutated status because such data were not available to patients or treating physicians.

In summary, our study suggests that PIK3CA mutation is associated with poor prognosis in resectable lung adenocarcinoma. This finding may have considerable clinical implications. Considerable effort has been focused on identifying therapeutic inhibitors of the PI3K/AKT pathway, in lung adenocarcinoma as well as in other common malignancies. Future studies are needed to confirm the association between PIK3CA mutations and the PI3K/AKT pathway and as well, to elucidate the exact mechanisms by which PIK3CA mutations affect tumor behavior. Thus, advances in the understanding of the molecular mechanisms of lung adenocarcinoma will lead to the development of novel and individualized anticancer therapeutic strategies.

\section{Disclosure}

The authors report no conflicts of interest in this work.

\section{References}

1. Jemal A, Bray F, Center MM, Ferlay J, Ward E, Forman D. Global cancer statistics. CA Cancer J Clin. 2011;61(2):69-90.

2. Alvarez M, Roman E, Santos ES, Raez LE. New targets for non-smallcell lung cancer therapy. Expert Rev Anticancer Ther. 2007;7(10): 1423-1437.

3. Hiles ID, Otsu M, Volinia S, et al. Phosphatidylinositol 3-kinase: structure and expression of the $110 \mathrm{kd}$ catalytic subunit. Cell. 1992;70(3): 419-429.

4. Catalogue of Somatic Mutation in Cancer.[homepage on the Internet]. England: Sanger Institute.[updated 2012 Dec 06]. Available from: http:// www.cancer.sanger.ac.uk/cosmic/gene/analysis? $\mathrm{ln}=P I K 3 C A$. Accessed March 29, 2013.

5. Okudela K, Suzuki M, Kageyama S, et al. PIK3CA mutation and amplification in human lung cancer. Pathol Int. 2007;57(10):664-6671.

6. An SJ, Chen $\mathrm{ZH}$, Su J, et al. Identification of enriched driver gene alterations in subgroups of non-small cell lung cancer patients based on histology and smoking status. PLoS One. 2012;7(6):e40109.

7. Chaft JE, Arcila ME, Paik PK, et al. Coexistence of PIK3CA and other oncogene mutations in lung adenocarcinoma-rationale for comprehensive mutation profiling. Mol Cancer Ther. 2012;11(2):485-491.

8. Board RE, Thelwell NJ, Ravetto PF, et al. Multiplexed assays for detection of mutations in PIK3CA. Clin Chem. 2008;54(4):757-760.

9. Spoerke JM, O’Brien C, Huw L, et al. Phosphoinositide 3-kinase (PI3K) pathway alterations are associated with histologic subtypes and are predictive of sensitivity to PI3K inhibitors in lung cancer preclinical models. Clin Cancer Res. 2012;18(24):6771-6783.

10. Lu HY, Su D, Pan XD, Jiang H, Ma SL. Mutation and expression of multiple treatment response-related genes in a population with locally advanced non-small cell lung cancer. Oncol Lett. 2012;3(2): 415-420.

11. Samuels Y, Ericson K. Oncogenic PI3K and its role in cancer. Curr Opin Oncol. 2006;18(1):77-82.

12. Zhao L, Vogt PK. Class I PI3K in oncogenic cellular transformation. Oncogene. 2008;27(41):5486-5496.

13. Kang S, Bader AG, Vogt PK. Phosphatidylinositol 3-kinase mutations identified in human cancer are oncogenic. Proc Natl Acad Sci USA. 2005;102(3):802-807.

14. Engelman JA, Chen L, Tan X, et al. Effective use of PI3K and MEK inhibitors to treat mutant Kras G12D and PIK3CA H1047R murine lung cancers. Nat Med. 2008;14(12):1351-1356.

15. Yamaguchi H, Yoshida S, Muroi E, et al. Phosphoinositide 3-kinase signaling pathway mediated by $\mathrm{p} 110 \alpha$ regulates invadopodia formation. J Cell Biol. 2011;193(7):1275-1288.

16. Baba Y, Nosho K, Shima K, et al. Phosphorylated AKT expression is associated with PIK3CA mutation, low stage, and favorable outcome in 717 colorectal cancers. Cancer. 2011;177(7):1399-1408. 
17. Liao $\mathrm{X}$, Lochhead $\mathrm{P}$, Nishihara R, et al. Aspirin use, tumor $P I K 3 C A$ mutation, and colorectal-cancer survival. NEngl J Med. 2012;367(17): 1596-1606.

18. Ogino S, Nosho K, Kirkner GJ, et al. PIK3CA mutation is associated with poor prognosis among patients with curatively resected colon cancer. J Clin Oncol. 2009;27(9):1477-1484.

19. Mangone FR, Bobrovnitchaia IG, Salaorni S, Manuli E, Nagai MA. $P I K 3 C A$ exon 20 mutations are associated with poor prognosis in breast cancer patients. Clinics (Sao Paulo). 2012;67(11):1285-1290.
20. Liao X, Morikawa T, Lochhead P, et al. Prognostic role of PIK3CA mutation in colorectal cancer: cohort study and literature review. Clin Cancer Res. 2012;18(8):2257-2268.

21. Fariña Sarasqueta A, Zeestraten EC, van Wezel T, et al. PIK3CA kinase domain mutation identifies a subgroup of stage III colon cancer patients with poor prognosis. Cell Oncol (Dordr). 2011;34(6):523-531.

\section{Publish your work in this journal}

OncoTargets and Therapy is an international, peer-reviewed, open access journal focusing on the pathological basis of all cancers, potential targets for therapy and treatment protocols employed to improve the management of cancer patients. The journal also focuses on the impact of management programs and new therapeutic agents and protocols on

\section{Dovepress}

patient perspectives such as quality of life, adherence and satisfaction. The manuscript management system is completely online and includes a very quick and fair peer-review system, which is all easy to use. Visit http://www.dovepress.com/testimonials.php to read real quotes from published authors.

\footnotetext{
Submit your manuscript here: http://www.dovepress.com/oncotargets-and-therapy-journal
} 